\title{
Le projet HANS KAII : une approche communautaire visant à améliorer la santé et le bien-être grâce au soutien par les pairs
}

\author{
Alexandra Henteleff, M. Éd.; Helena Wall, M. Éd. \\ Cet article de recherche par méthodes mixtes originale a fait l'objet d'une évaluation par les pairs.
}

Diffuser cet article sur Twitter

\begin{abstract}
Résumé
Introduction : Le modèle HANS KAÏ est une intervention de promotion de la santé originale qui vise à améliorer la santé des participants en ciblant les comportements interdépendants de prévention des maladies chroniques grâce au soutien par les pairs et au renforcement des réseaux de soutien social. L'objectif de notre étude était de mesurer l'efficacité du modèle HANS KAÏ en contexte urbain canadien.
\end{abstract}

Méthodologie : Nous avons utilisé un modèle de recherche interventionnelle à méthodes mixtes appliqué à plusieurs sites entre novembre 2010 et avril 2015. Les données ont été recueillies au moyen de sondages auprès des participants et d'entrevues en personne au début de l'étude puis à 6, 12 et 24 mois. Les participants se sont réunis en groupes au moins une fois par mois durant la période de recherche afin de surveiller eux-mêmes leurs indicateurs de santé, de préparer et de partager une collation saine, de participer à une activité physique, d'établir un objectif en matière de mode de vie sain (facultatif) et d'avoir une activité de convivialité.

Résultats : Des améliorations statistiquement significatives de la santé mentale ont été constatées avant et après le programme, et $66 \%$ des participants ont fait état de changements de comportement précis résultant de la participation au projet HANS. D’autres effets positifs sur la santé ont été constatés : un soutien par les pairs, l'acquisition de connaissances précises en matière de santé, l'inspiration, la motivation et la prise de responsabilité, l'autonomisation découlant du suivi de ses propres indicateurs de santé, la possibilité de rompre l'isolement social et enfin une meilleure connaissance de la façon d'avoir accès aux services.

Conclusion : C'est la nécessité de trouver des moyens innovants pour favoriser la prévention et la gestion des maladies chroniques qui a motivé la mise en œuvre et l'évaluation du modèle HANS KAII. Bien que d'autres recherches soient nécessaires pour valider nos résultats, il semble que le modèle HANS KAÏ puisse être efficace pour créer un contexte permettant à la population locale de s'entraider en favorisant des choix de vie sains et en détectant de façon précoce les changements d'état de santé.

Mots-clés : promotion de la santé, maladie chronique, prévention, soutien social, groupe de pairs, groupes d'entraide, soutien par les pairs

\section{Introduction}

Cet article décrit une intervention communautaire novatrice de promotion de la santé offerte par une coopérative de soins de santé du Manitoba, qui a permis d'étudier la relation entre le soutien par les pairs et les perceptions du mieux-être. Le soutien par les pairs est défini comme « un système par lequel on donne et on reçoit de l'aide en s'inspirant des principes fondamentaux que sont le respect, la responsabilité partagée et l'accord mutuel quant à ce qui est utile »[traduction] $]^{1, p .137}$. On vise à comprendre avec empathie la situation d'une autre personne par le biais de l'expérience commune. En établissant une relation de confiance, on peut se remettre en question avec respect, essayer d'adopter avec les

\section{Points saillants}

- Nous avons besoin de méthodes novatrices pour prévenir et gérer les maladies chroniques au sein de populations défavorisées sur le plan socioéconomique.

- Le modèle HANS KAİ fait appel au soutien par les pairs pour amener la population locale à se soutenir mutuellement en encourageant des choix de vie sains, en misant sur des comportements de prévention des maladies chroniques et en aidant à détecter précocement des changements d'état de santé.

- L'originalité du modèle HANS KAİ provient du fait qu'il cible la santé, alors que la plupart des interventions de soutien par les pairs visent une maladie.

- La participation au projet HANS KAII a entraîné une amélioration statistiquement significative des scores en santé mentale.

- Les participants ont également signalé une diminution de leur isolement social, l'adoption de comportements sains, une meilleure connaissance des services, une augmentation de l'utilisation des services et une autonomisation découlant du suivi de leurs indicateurs de santé.

autres de nouveaux comportements et modifier la représentation qu'on se fait de soi-même. C'est ce qu'on appelle l'autonomisation mutuelle ${ }^{1}$.

Le soutien par les pairs a été utilisé pour aider les individus à s'adapter à des changements de vie tels que la naissance d'un enfant, des pertes importantes, des incapacités à long terme ou des maladies

Rattachement des auteures :

Infirmières de recherche affiliées en milieu communautaire, Université du Manitoba, Winnipeg (Manitoba), Canada

Correspondance : Alexandra Henteleff, infirmière de recherche affiliée en milieu communautaire, Université du Manitoba, 1469, Wellington Cres South, Winnipeg (Manitoba) R3N 0B6; tél. : 204-793-8759; courriel : ahenteleff@gmail.com 
chroniques et, en matière d'initiatives de promotion de la santé, particulièrement le soutien pour aider à modifier les comportements en matière de santée ${ }^{2-4}$. L'étude de Ford et de ses collaborateurs indique que les programmes de soutien par les pairs " apparaissent comme des moyens très efficaces et habilitants, qui permettent aux gens de gérer leurs problèmes de santé »

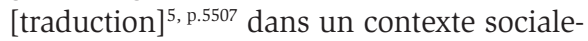
ment favorable. Nous n'avons trouvé dans la littérature aucune description d'initiative locale canadienne non axée sur une maladie en particulier et ayant utilisé un modèle de soutien par les pairs pour créer des réseaux sociaux en soutien au mieux-être personnel.

\section{Intervention}

Le modèle HANS KAÏ est une intervention originale de promotion de la santé qui vise à améliorer la santé des participants grâce au soutien par les pairs et au renforcement des réseaux de soutien social. Le projet HANS KAII s'inspire du han, un style de gestion de la santé instauré par des coopératives de santé japonaises au milieu des années 1950 pour encourager les gens à prendre en main leur santé et pour promouvoir la médecine préventive ${ }^{6}$. En japonais, le mot han signifie " groupe ", et le terme kaï signifie " assemblée » ou " réunion ». L'approche de groupe han visait à alléger le fardeau des soins primaires et à encourager le soutien par les pairs et l'autonomisation personnelle ${ }^{6}$. Les groupes HANS KAÏ (groupes HANS) sont généralement composés d'environ 10 membres rassemblés sur la base de caractéristiques communes comme l'âge, le secteur de résidence et la disponibilité. L'idée que les personnes qui passent du temps ensemble et surveillent leur santé vivront plus longtemps et en meilleure santé est constitutive de cette intervention? .

La théorie de l'apprentissage social ${ }^{8}$ et la théorie de l'autonomisation ${ }^{9}$ constituent les bases théoriques de l'intervention HANS KAII. La théorie de l'apprentissage social explique le comportement humain comme une interaction permanente entre les influences cognitives, comportementales et environnementales : on apprend en observant les comportements et les attitudes des autres, ainsi que les effets de ces comportements $^{7}$. Comme la théorie de l'apprentissage social de Bandura ${ }^{8}$, l'autonomisation inclut le concept d'auto-efficacité, selon lequel les sentiments de compétence, de contrôle personnel et d'image de soi positive aident les individus à concevoir positivement leur capacité à changer et à maîtriser les problèmes ${ }^{9}$. L'approche de l'autonomisation "redéfinit le rôle du professionnel [...] en rôle de collaborateur [...] [où] les participants jouent un rôle actif dans le processus de changement $»^{9, p .44-45}$.

Notre revue de la littérature n’a détecté aucune recherche sur l'efficacité des groupes de soutien par les pairs pour améliorer la santé globale des participants. Nous avons entrepris ce projet de recherche pour mettre en œuvre le modèle HANS KAÏ et évaluer son efficacité en milieu urbain canadien. Dans notre modèle, les pairs des groupes HANS étaient les membres du groupe. Les participants ont eu au moment de leur inscription à s'inscrire à une « école de la santé » afin d'acquérir des connaissances de base sur un ensemble de sujets relatifs à la santé ainsi qu'une formation sur la façon de prendre leurs mesures personnelles de santé et de travailler de façon autonome en tant que groupe. Les participants se sont ensuite réunis en petits groupes au moins une fois par mois pendant environ deux heures pour s'informer mutuellement sur des sujets de santé et se renseigner auprès de fournisseurs de soins, pour participer à des activités physiques, pour préparer et partager une collation nutritive, pour surveiller et consigner leurs mesures de santé dans leurs journaux de bord, et enfin pour prendre du temps de convivialité.

\section{Contexte}

L’organisme NorWest Co-op Community Health (NorWest) vise à rassembler la population locale dans une démarche coopérative de santé et de mieux-être en cherchant à ce que chacun prenne en charge sa santé. NorWest est situé dans la communauté Inkster de Winnipeg, un secteur défavorisé sur le plan socioéconomique, où $20 \%$ des familles vivent sous le seuil de faible revenu ${ }^{10}$. Le secteur manque d'installations récréatives et, d'après un rapport de $2008^{11}, 86 \%$ des résidents estimaient que le soutien social était essentiel pour changer les comportements liés à l'alimentation et à l'activité physique, et qu'il s'agissait du facteur le plus important pour adhérer à des programmes de vie saine. Le modèle HANS KAÏ rejoignant la vision, la mission et les valeurs de NorWest, l'organisme a voulu vérifier si la mise en œuvre du modèle était possible et bénéfique.

\section{Objectifs de la recherche}

Le but de cette étude était de déterminer si des groupes locaux structurés et dirigés par des pairs pouvaient être instaurés avec succès en contexte urbain canadien et avaient un effet positif sur la santé et le bien-être des participants. Les objectifs de l'intervention étaient les suivants :

1. créer des réseaux de soutien social et de soutien par les pairs pour favoriser le mieux-être des participants en accroissant leur capacité à faire des choix de vie plus sains, donner aux participants des moyens d'améliorer leur santé et leur permettre de tisser des liens afin de réduire l'isolement social;

2. rendre les participants davantage conscients du lien entre mieux-être personnel, choix de vie sains, poids santé et facteurs sur lesquels on peut ou non agir;

3. maintenir ou améliorer des indicateurs de santé mesurables;

4. accroître l'accès aux soins primaires et à d'autres programmes ou services communautaires.

\section{Méthodologie}

\section{Conception de la recherche}

Nous avons utilisé un modèle participatif faisant appel à la population locale à chaque étape de la recherche. Un conseil local a sollicité les services d'un chercheur par l'entremise d'une demande de manifestation d'intérêt afin d'étudier les répercussions de la participation à un groupe HANS sur un ensemble de résultats en matière de santé. Il a examiné les réponses reçues, a sélectionné et rencontré l'équipe de recherche et a contribué à la conception finale. Le personnel de NorWest était surtout responsable de soutenir la mise en œuvre du projet HANS et de travailler en étroite collaboration avec les chercheurs lors du déroulement de l'étude. Des membres de groupes locaux existants ont participé aux essais du modèle HANS KAÏ et ont fourni des commentaires précieux, ce qui a permis de peaufiner les outils de recherche. Les résultats préliminaires ont été communiqués lors de présentations au conseil local et aux groupes HANS ainsi qu'à des événements communautaires et à l'assemblée générale annuelle de NorWest en juin 2017. 
Nous avons utilisé un modèle de recherche interventionnelle à méthodes mixtes appliqué à plusieurs sites. Cette forme d'enquête flexible a permis de saisir les divers points de vue et a favorisé une compréhension plus complète de l'expérience de l'intervention $^{12}$.

Les données quantitatives mesurées avant et après l'intervention proviennent de sondages auprès des participants et ont été saisies dans une base de données conçue spécialement à cet effet. Les observations qualitatives ont été obtenues grâce à des entrevues individuelles avec les participants. La collecte de données a eu lieu entre novembre 2010 et avril 2015. Le sondage et les entrevues ont été effectués au départ (référence, 0) et à 6, 12 et 24 mois. L'étude a reçu l'approbation écrite du Conseil d'éthique de la recherche en éducation et en soins infirmiers de l'Université du Manitoba, protocole n E2010:102.

\section{Recrutement}

Des techniques de recrutement variées ont été utilisées : des présentations locales ou en milieu de travail, des envois postaux aux clients de NorWest, des recommandations de fournisseurs de soins de santé, des affiches situées dans des lieux publics (épiceries, pharmacies, centres communautaires et cliniques médicales) et le recrutement en personne par des présentations sur le projet HANS à des événements et des rassemblements locaux. Les critères d'inclusion étaient les suivants : être résident des secteurs Inkster ou Seven Oaks de Winnipeg, avoir au moins 18 ans, être capable de parler et de lire l'anglais et être en relativement bonne santé. Les personnes atteintes de maladies chroniques étaient admissibles dans la mesure où leur état de santé était stable.

Une approche spécifique a été utilisée pour recruter les participants isolés socialement ou défavorisés économiquement, comme les aînés, les nouvelles mères et les néoCanadiens. Après avoir rempli le formulaire obligatoire de demande, les personnes souhaitant participer au projet ont été réparties dans les groupes préexistants ou dans de nouveaux groupes en fonction de leur âge, de leur lieu de résidence et de leur disponibilité.

Les participants ont suivi la formation " école de la santé » HANS composée de six séances de deux heures en présentiel destinées à leur apprendre comment surveiller leur santé et travailler de façon autonome en tant que groupe. Les sessions ont été articulées autour du principe que de nombreux facteurs influent sur la santé. Les sujets abordés ont été les indicateurs de santé, les maladies chroniques, l'alimentation, l'activité physique, le sommeil, le stress, la santé générale, les soins primaires au fil des ans, les médicaments, les suppléments alimentaires, le tabagisme, les soutiens sociaux et la façon de travailler efficacement en groupe. Les participants devaient obligatoirement suivre les cours information, les mêmes connaissances et le même engagement.

Les groupes HANS, composés de 8 à 15 perles participants, avec un soutien et des conseils fournis au besoin par les professionnels de la santé de NorWest. Les problèmes potentiels d'accès ont été pris en compte au moment de la recherche, et des mesures ont été prises pour atténuer les obstacles économiques à la participation, par exemple en offrant des services de garde d'enfants ou des séances d'entraînement gratuites et en encourageant le covoiturage. Les groupes se sont réunis au moins une fois par mois, à raison de 1,5 à 2 heures par séance. Certains se sont réunis plus souvent. Chaque séance comprenait obligatoirement les composantes suivants : 1) surveiller les indicateurs de santé; 2) préparer une collation santé; 3) participer à une activité physique; 4) présenter un plan d'action pour le mois (fixer un objectif de mode de vie) - facultatif; 5) échanger ses coordonnées avec un " ami » pour des contacts réguliers et 6) entrer en relation avec d'autres personnes. sur la santé pour que tous aient la même sonnes, ont été conçus pour être dirigés par

$\mathrm{Au}$ cours des trois ans de recrutement, treize groupes ont démarré. Certains ont réussi à se réunir régulièrement tout au long de la période de recherche alors que d'autres ne se sont réunis que brièvement et n’ont pas réussi à atteindre la cohésion nécessaire pour poursuivre leurs rencontres. Neuf groupes (soit 77 personnes) ont été inclus dans cette recherche. Pour être retenus, les groupes devaient s'être réunis régulièrement et avoir participé à au moins trois périodes de collecte de données prescrites $(0,6,12,24$ mois). Sept groupes se sont réunis dans des espaces communautaires et deux groupes en milieu de travail. La participation aux entrevues en personne s'est révélée variable d'un groupe à l'autre (tableau 1).

Le personnel de NorWest a proposé plusieurs raisons à l'origine des abandons : manque pour l'individu d'adéquation au groupe, caractéristiques sociodémographiques, retour au travail, contraintes du lieu de travail, changements dans les besoins de l'individu, stress familial causé par le manque de temps, départ du participant après obtention de ce dont il avait besoin et enfin déménagement.

\section{Collecte de données}

Les données ont été collectées à l'aide d'un sondage auprès des participants et d'entrevues individuelles en face à face. Les journaux de bord des participants destinés au suivi de certains indicateurs de santé n'ont pas été utilisés de manière égale et n'ont donc pas fourni suffisamment de données quantitatives pour l'analyse.

TABLEAU 1

Entrevues en personne auprès des participants HANS KAÏ (2010-2015)

\begin{tabular}{lccc} 
& Type de groupe & Effectif & $\begin{array}{c}\text { Membres ayant participé à } \\
\text { au moins 3 collectes de données }\end{array}$ \\
\hline Groupe 1 & Local & 5 & 5 \\
Groupe 2 & Local & 7 & 3 \\
Groupe 3 & Local & 12 & 4 \\
Groupe 4 & Local & 12 & 7 \\
Groupe 5 & Milieu de travail & 9 & 8 \\
Groupe 6 & Local & 5 & 5 \\
Groupe 7 & Local & 7 & 6 \\
Groupe 8 & Milieu de travail & 10 & 8 \\
Groupe 9 & Local & 10 & 53 \\
\hline Total & & 77 & 53 \\
\hline
\end{tabular}




\section{Sondage auprès des participants HANS}

À partir d'outils validés provenant de différentes sources ${ }^{13-19}$, nous avons créé un questionnaire complet spécifiquement pour cette étude afin d'évaluer les effets de la participation aux groupes HANS sur les thèmes liés à la santé suivants :

- connaissance du diabète et de l'hypertension;

- autoévaluations de l'alimentation, de l'activité physique et du sommeil;

- tabagisme;

- état de santé mentale;

- accès à des fournisseurs de soins de santé;

- connaissance des programmes et des services communautaires;

- compréhension de la façon d'améliorer son état de santé général;

- liens avec les habitants du quartier et de la ville.

Le questionnaire du projet, élaboré pour maximiser la validité et la fiabilité du contenu, a été révisé et modifié par un groupe d'experts multidisciplinaire jouissant d'une expérience et d'une expertise en mobilisation communautaire et en prestation de services.
Le personnel de NorWest a distribué et recueilli les questionnaires remplis, à savoir les sondages initiaux remplis par les participants lors de leur première séance à l'école de la santé (entrevue 1), ainsi que les sondages de suivi à 6,12 et 24 mois. Les données ont été saisies dans une base de données par le personnel de soutien de NorWest.

\section{Entrevues individuelles}

Les chercheurs ont interrogé tous les participants au début de l'intervention (entrevue initiale) puis à l'occasion des entrevues de suivi à 6, 12 et 24 mois. Dans l'entrevue initiale, deux questions portaient sur la perception des participants quant aux facteurs ayant un effet positif ou négatif sur leur santé. Les entrevues de suivi comportaient, outre ces deux questions, diverses questions visant à recueillir des commentaires sur l'effet perçu de la participation régulière à un groupe HANS et sur les moyens d'améliorer le projet HANS KAÏ. Pour la première entrevue, les enquêteurs ont pris des notes sur papier puis les ont retranscrites, alors que les entrevues de suivi ont été quant à elles enregistrées puis retranscrites. Les questions et le calendrier des entrevues sont présentés dans le tableau 2.

Les entrevues initiales ont eu lieu lors de la première réunion de chaque groupe HANS après sa création. Les entrevues de suivi

TABLEAU 2

Questions des entrevues individuelles pour les participants HANS

\begin{tabular}{|c|c|c|}
\hline Question & $\begin{array}{c}\text { Entrevues } \\
\text { initiales }^{\mathrm{a}}\end{array}$ & Entrevues de suivi ${ }^{\mathrm{b}}$ \\
\hline $\begin{array}{l}\text { 1. Au cours des } 6 \text { derniers mois, qu'est-ce qui vous a aidé à } \\
\text { rester en santé et à vous sentir bien? }\end{array}$ & $\checkmark$ & $\checkmark$ \\
\hline $\begin{array}{l}\text { 2. Au cours des } 6 \text { derniers mois, qu'est-ce qui ne vous a pas } \\
\text { aidé ou a nui à votre capacité à rester en santé et à vous sentir } \\
\text { bien? }\end{array}$ & $\checkmark$ & $\checkmark$ \\
\hline $\begin{array}{l}\text { 3. Depuis que vous avez rejoint le groupe HANS, avez-vous } \\
\text { reçu le soutien d'un membre du groupe? Dans l'affirmative, } \\
\text { veuillez décrire la situation. }\end{array}$ & & $\checkmark$ \\
\hline $\begin{array}{l}\text { 4. Depuis que vous avez rejoint le groupe HANS, avez-vous } \\
\text { apporté votre soutien à un membre du groupe? Dans } \\
\text { l'affirmative, veuillez décrire la situation. }\end{array}$ & & $\checkmark$ \\
\hline $\begin{array}{l}\text { 5. En quoi le fait de faire partie d'un groupe HANS vous a-t-il } \\
\text { aidé à améliorer votre santé ou à rester en bonne santé? }\end{array}$ & & $\checkmark$ \\
\hline 6. Qu'avez-vous le plus aimé dans le programme HANS? & & $\checkmark$ \\
\hline 7. Qu'avez-vous moins aimé du programme HANS? & & $\checkmark$ \\
\hline 8. Comment pourrait-on améliorer le programme? & & $\checkmark$ \\
\hline
\end{tabular}

Entrevues initiales (avant l'intervention).

${ }^{b}$ Entrevues de suivi à 6, 12 et 24 mois. avaient été prévues à 6 et 12 mois après le début de l'étude mais, comme tous les participants n'ont pu être interrogés à ces intervalles en raison de l'absence de certains et de l'impossibilité d'organiser certaines réunions, les chercheurs n'ont pas été en mesure d'interroger la plupart des participants aux trois intervalles prévus. Un suivi des réponses a été effectué pour les 53 personnes qui ont participé à l'entrevue initiale (entrevue 1, de référence) et à au moins deux entrevues de suivi (entrevues 2 et 3 ) à 6,12 ou 24 mois.

\section{Analyse des données}

\section{Sondage}

Nous avons utilisé un test $t$ pour conduire notre analyse avant/après des données de sondage, avec un seuil alpha (ou $p$ ) fixé à 0,05 . Nous avons adapté l'approche en intention de traiter (ITT) souvent utilisée dans les essais cliniques ${ }^{20}$. Nous avons utilisé un test $z$ pour comparer les caractéristiques individuelles des participants ayant terminé le projet et de ceux ne l'ayant pas terminé ou qui ont été perdus de vue.

\section{Entrevues}

Nous avons effectué une analyse thématique des réponses d'entrevue. Les chercheurs principaux ont analysé séparément les transcriptions des données des 48 premières entrevues initiales et des 24 premières entrevues de suivi. Ils ont ensuite généré des codes initiaux pour rechercher, définir et nommer des thèmes, ce qui a conduit à un canevas de codage. Un chercheur principal et un assistant de recherche ont ensuite revu et codé de façon indépendante toutes les transcriptions, ont discuté de leurs analyses respectives et se sont mis d'accord sur l'interprétation des données.

\section{Résultats}

\section{Caractéristiques individuelles des participants}

Les caractéristiques individuelles des participants sont celles de 63 personnes, cependant pas toutes les personnes ont répondu à chacune des questions. Les participants étaient principalement des femmes (60 sur 63) et étaient âgés de 20 à 72 ans (figure 1). En matière d'état matrimonial, on peut séparer les participants ayant répondu à cette question en deux groupes d'égale importance : ceux mariés ou en union libre (30 sur 63) et les célibataires, 
veufs, divorcés ou séparés (30 sur 63). Trente-sept pour cent (23 sur 63) avaient des enfants à la maison, 52 \% (33 sur 63) n'avaient aucun enfant à la maison et la moitié (28 sur 63) vivaient seuls. Trentecinq pour cent avaient fait des études collégiales ou universitaires et $41 \%$ avaient un diplôme d'études secondaires. Près du tiers des participants vivaient dans leur localité depuis au moins 25 ans (figure 2). Plus du tiers étaient sans emploi (figure 3) et un nombre similaire de participants ont déclaré un revenu annuel inférieur à 40000 \$ (figure 4), sachant néanmoins que $38 \%$ [24 sur 63] des participants n'ont pas répondu à cette dernière question.

Le test $z$ ayant servi à comparer les caractéristiques individuelles a révélé des différences statistiquement significatives entre les participants ayant terminé le projet et les autres. Ceux qui ont été perdus de vue étaient moins instruits, présentaient un taux de chômage plus élevé et avaient des revenus plus faibles.

\section{Réponses concernant les mesures liées à la santé avant et après le programme}

La principale conclusion de l'analyse des sondages avant et après l'intervention (tableau 3) est que les seules améliorations statistiquement significatives à la suite du programme relevaient du score relatif au continuum de la santé mentale, qui visait à évaluer le bien-être et considérait la santé mentale comme étant "florissante ", " relativement bonne » ou « languissante $»^{18}$.

Les autres mesures liées à la santé ne présentaient pas d'amélioration statistiquement significative à la suite du programme.

\section{Résultats des entrevues individuelles : facteurs influençant la santé}

Les participants devaient nommer les facteurs ayant eu un effet positif ou négatif sur leur santé au cours des six mois précédents. Les réponses ont été les mêmes tout au long de la période de collecte des données, si ce n'est qu'à 6,12 et 24 mois, davantage de participants ont fait état d'un effet positif de la participation au groupe HANS.

\section{Facteurs positifs influençant la santé}

Sept facteurs ont été mentionnés comme ayant un effet positif sur la santé. Les relations de soutien avec la famille et les amis étaient le facteur le plus fréquemment mentionné pour les entrevues initiales comme de suivi. Le soutien des autres membres du groupe HANS a également été mentionné. L'activité physique, que ce soient les activités organisées ou les activités de loisirs, ainsi que l'amélioration de l'alimentation ont été systématiquement mentionnées au cours des entrevues comme des facteurs améliorant la santé. Divers soutiens communautaires ont été mentionnés au début (entrevue initiale) puis, au cours des entrevues suivantes, la participation au groupe HANS a constitué la réponse la plus fréquente concernant le soutien communautaire. Les participants ont également indiqué qu'une bonne santé mentale avait un impact positif sur l'état de santé général, en particulier sur le fait d'être heureux au travail et moins stressé, de même que le fait d'avoir une attitude et des croyances positives. Un plus grand nombre de participants au fil du temps ont mentionné l'effet positif de l'accès aux services et, en particulier, aux services de NorWest.

\section{Facteurs négatifs influençant la santé}

Six facteurs ont été mentionnés comme ayant un effet négatif sur la santé. Une mauvaise santé mentale et le stress ont été le plus souvent cités comme affectant la santé globale. Les participants ont déclaré se sentir dépassés et vivre du stress, que ce soit au travail, en famille ou ailleurs. Cependant, à la période 3 , moins de participants ont mentionné qu'une mauvaise santé mentale ou le stress avait un effet négatif sur leur santé. De nombreux participants ont indiqué qu'une mauvaise alimentation avait un impact négatif, en particulier un environnement social qui contribue à accroître la consommation de nourriture, les mauvais choix alimentaires et les difficultés à maintenir le poids ou à prendre du poids. Cependant, à la période 3 , moins de participants ont mentionné que la mauvaise alimentation avait un effet négatif sur leur santé. Le manque d'activité physique, une mauvaise santé, le manque de temps et d'équilibre entre le travail et la vie personnelle et les relations non favorables ont également été mentionnés comme étant des facteurs négatifs dans les entrevues initiales comme de suivi. Le tableau 4 regroupe par thème les facteurs qui ont eu des effets positifs ou négatifs sur la santé, avec des citations représentatives de chaque catégorie.

\section{Résultats des entrevues individuelles : effet} positif de la participation au groupe HANS

Les questions 3 à 6 visaient à déterminer si la participation au groupe HANS avait eu
FIGURE 1

Âge des participants au projet HANS

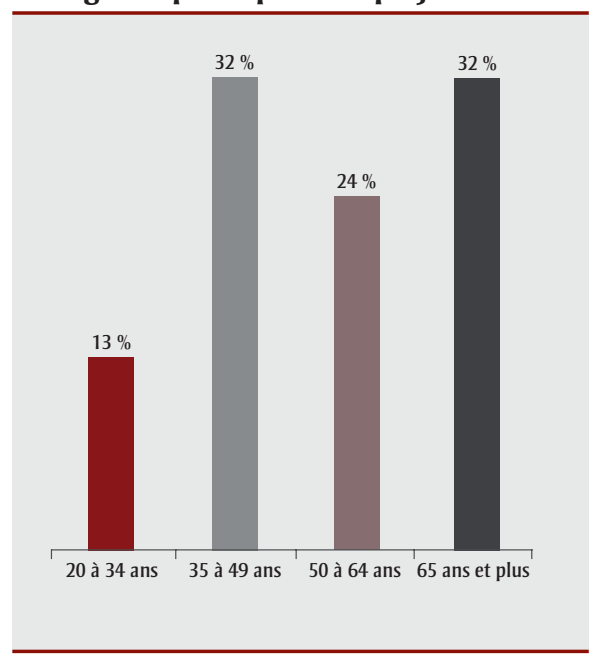

FIGURE 2

Nombre d'années depuis lesquelles les participants au projet HANS vivent dans leur localité

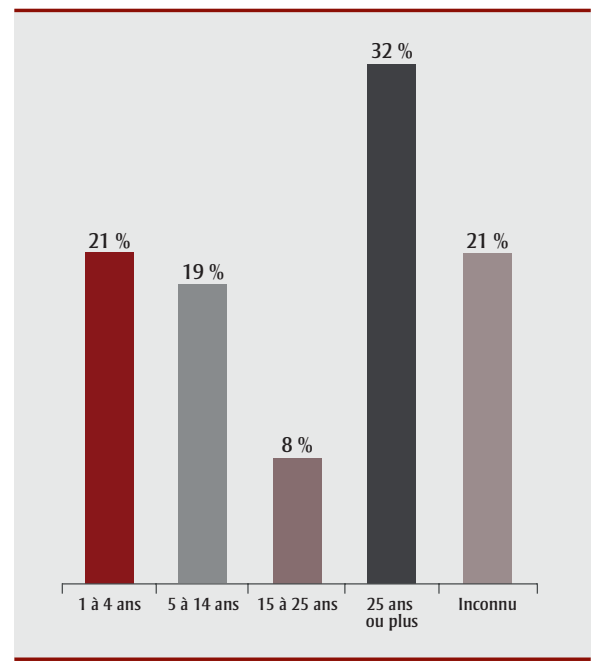

FIGURE 3

Statut professionnel des participants au projet HANS

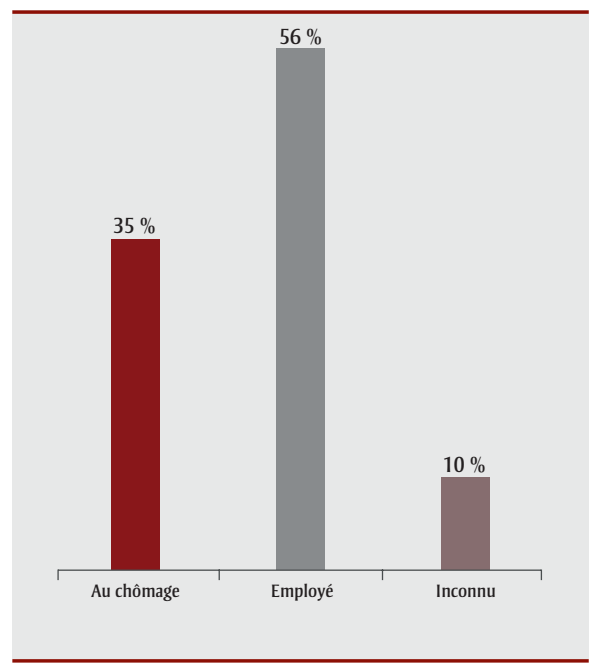


FIGURE 4

Revenu annuel du ménage des participants au projet HANS

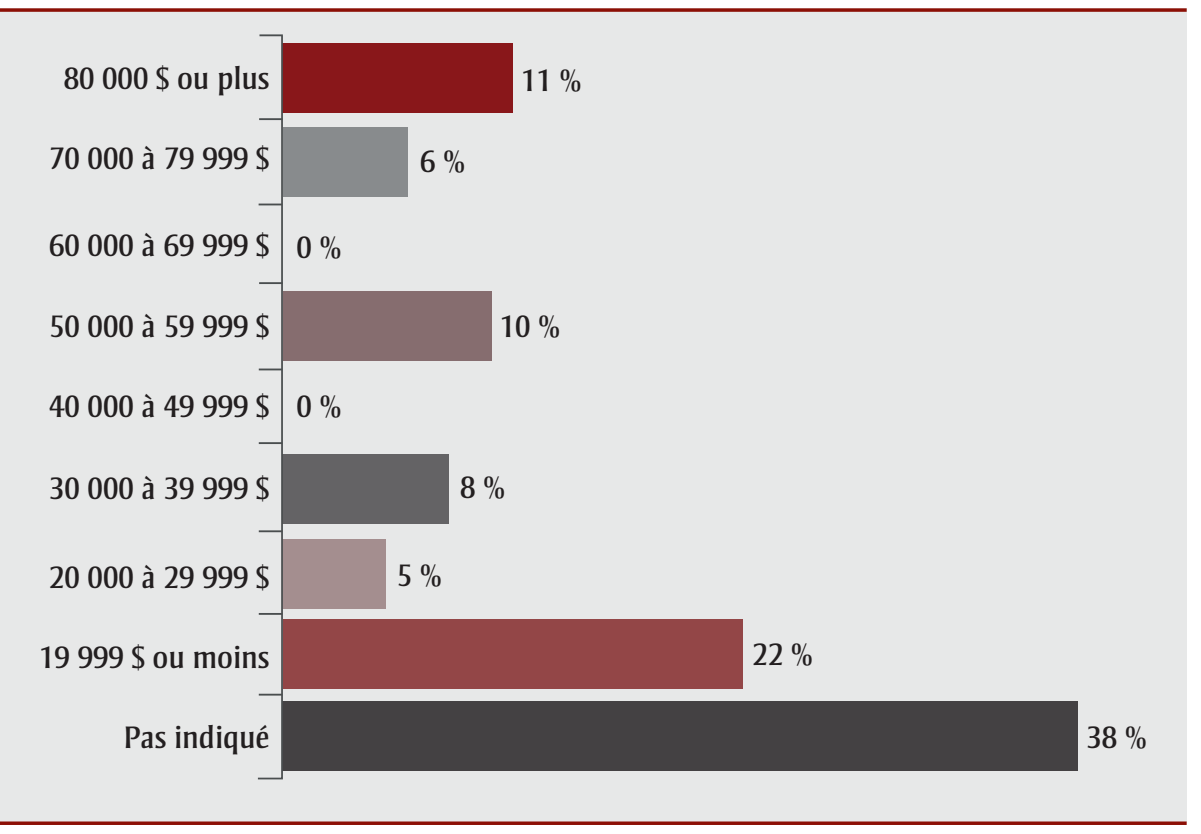

un effet positif sur la santé des participants et ce que les participants aimaient du modèle HANS. Les différents thèmes qui sont ressortis de notre analyse et leur distribution sont représentés sur la figure 5 et sont décrits plus en détail ci-dessous.

\section{Soutien par les pairs}

Les réponses les plus fréquentes concernant les avantages et l'effet de la participation à un groupe HANS relèvent du soutien par les pairs. Une phrase souvent entendue a été "nous nous soutenons tous les uns les autres ». Les participants ont dit s'écouter et se soutenir mutuellement, exprimer leurs problèmes, offrir du soutien au cours d'une situation difficile de la vie et avoir un sentiment de sécurité et d'absence de jugement. Ils ont également fait état de nouveaux amis et d'un sentiment d'appartenance découlant de la participation au groupe HANS. Une participante a mentionné comment elle avait soutenu une autre participante dont l'enfant était victime de harcèlement à l'école et l'avait aidée à « trouver une solution ». Certains participants ont indiqué faire du covoiturage pour se rendre aux réunions du groupe ou à des rendez-vous médicaux, ou encore présenter aux autres les ressources qu'ils jugeaient utiles. D'autres ont dit avoir offert leur aide à une autre personne en dehors de la réunion de groupe et être restés en contact entre les réunions grâce à des appels téléphoniques réguliers, des courriels, des messages, des promenades ou des sorties pour boire un café.

\section{Apprentissage et connaissances}

Les participants ont décrit la manière dont ils ont reçu l'information en matière de santé et de choix sains à la fois dans la formation d'école de la santé, de la part de conférenciers invités et entre participants. Ils ont fait état de l'impact de leurs nouvelles compétences, que ce soit par des exercices sur chaise, des mesures liées à l'état de santé (p. ex. glycémie ou pression artérielle), la Zumba, la cuisine ou la méditation.

\section{Changement de comportement} état de changements de comportement précis résultant de leur participation au groupe HANS. Ces changements concernaient principalement l'alimentation et l'activité physique, ainsi que d'autres domaines comme la gestion du stress, du poids, de la glycémie ou de la pression artérielle. Les participants ont également décrit les effets positifs de la fixation d'objectifs ainsi que d'autres changements. De plus, ils ont expliqué comment les apprentissages découlant du groupe HANS avaient eu des répercussions positives plus large, chez d'autres membres de leur famille.

\section{Inspiration, motivation et prise de responsabilité}

Les participants ont mentionné à quel point la participation à un groupe HANS avait amélioré leur santé par l'inspiration, la motivation ou la prise de responsabilité.
Les deux tiers des participants ont pu faire
Ils ont également fait état de leur désir de faire profiter les autres des avantages du groupe HANS, en encourageant d'autres personnes à s'y joindre ou en diffusant l'information reçue au sein du groupe.

\section{Indicateurs de suivi}

Certains participants ont déclaré que la surveillance de leurs indicateurs de santé dans un journal de bord était stimulante, rassurante et motivante. Ils ont aimé pouvoir être capables d'assurer eux-mêmes leur suivi, et le constat de ces mesures les a incités à demander un soutien médical au besoin (par exemple, " parce que j'ai découvert que je faisais de l'hypertension artérielle au sein du groupe HANS KAII, j'ai consulté mon médecin, et la situation est à présent sous contrôle »).

\section{Rompre l'isolement social}

Certains participants ont mentionné que la participation à un groupe HANS avait renforcé leur sentiment d'appartenance et les avait motivés à « sortir ».

\section{Accès aux services}

Plusieurs participants ont noté que leur participation au groupe HANS avait constitué une passerelle d'accès aux services grâce aux services fournis par NorWest, les avait encouragés à consulter régulièrement des professionnels de la santé et à s'informer sur d'autres services communautaires.

Le tableau 5 résume les répercussions positives sur la santé de la participation à un groupe HANS, avec des citations représentatives.

\section{Résultats des entrevues individuelles: recommandations pour améliorer les groupes HANS}

Les questions 7 et 8 visaient à recueillir les commentaires des participants sur la façon d'améliorer le modèle HANS. Les principaux points d'amélioration mentionnés sont présentés ci-dessous.

\section{Format de la réunion}

Les participants ont affirmé que la fréquence et la durée posaient problème, mais n'ont pas pu s'entendre sur une fréquence ou une durée optimales. Certains ont mentionné le besoin d'avoir des membres supplémentaires au sein de leur groupe. 
TABLEAU 3

Analyse des réponses des participants concernant les mesures liées à la santé avant et après le programme HANS

\begin{tabular}{|c|c|c|c|c|}
\hline \multirow[b]{2}{*}{ Mesure liée à la santé } & \multirow[b]{2}{*}{ Réponse } & \multicolumn{2}{|c|}{ Événement de collecte de données } & \multirow{2}{*}{$\begin{array}{l}p \\
\text { (pour la comparaison } \\
\text { de colonnes) }\end{array}$} \\
\hline & & Premier sondage (\%) & Dernier sondage (\%) & \\
\hline \multirow{2}{*}{$\begin{array}{l}\text { Score de connaissance sur le diabète } \\
(n=57)\end{array}$} & Score faible & 19,3 & 7,0 & $>0,05$ \\
\hline & Score élevé & 80,7 & 93,0 & $>0,05$ \\
\hline \multirow{2}{*}{$\begin{array}{l}\text { Score de connaissance sur l'hypertension } \\
(n=58)\end{array}$} & Score faible & 6,9 & 5,2 & $>0,05$ \\
\hline & Score élevé & 93,1 & 94,8 & $>0,05$ \\
\hline \multirow{3}{*}{$\begin{array}{l}\text { Score lié à l'alimentation } \\
(n=61)\end{array}$} & $\begin{array}{l}\text { Faible risque lié à } \\
\text { l'alimentation }\end{array}$ & 31,1 & 41,0 & $>0,05$ \\
\hline & $\begin{array}{l}\text { Risque modéré lié à } \\
\text { l'alimentation }\end{array}$ & 37,7 & 37,7 & $>0,05$ \\
\hline & $\begin{array}{l}\text { Risque élevé lié à } \\
\text { l'alimentation }\end{array}$ & 31,1 & 21,3 & $>0,05$ \\
\hline \multirow{5}{*}{$\begin{array}{l}\text { Score lié à l'activité physique } \\
(n=61)\end{array}$} & Très actif & 4,9 & 9,8 & $>0,05$ \\
\hline & Actif & 34,4 & 39,3 & $>0,05$ \\
\hline & Acceptable & 23,0 & 21,3 & $>0,05$ \\
\hline & Inactif & 13,1 & 13,1 & $>0,05$ \\
\hline & Sédentaire & 24,6 & 16,4 & $>0,05$ \\
\hline \multirow{2}{*}{$\begin{array}{l}\text { Échelle du sommeil } \\
(n=60)\end{array}$} & Pas de problème & 70,0 & 68,3 & $>0,05$ \\
\hline & Problématique & 30,0 & 31,7 & $>0,05$ \\
\hline \multirow{3}{*}{$\begin{array}{l}\text { Score du continuum de la santé mentale } \\
(n=60)\end{array}$} & Florissante & 43,3 & 63,3 & $<0,05$ \\
\hline & Languissante & 10,0 & 6,7 & $>0,05$ \\
\hline & Relativement bonne & 46,7 & 30,0 & $>0,05$ \\
\hline \multirow{2}{*}{$\begin{array}{l}\text { Avez-vous déjà fumé? } \\
(\mathrm{n}=60)\end{array}$} & Non & 55,0 & 58,3 & $>0,05$ \\
\hline & Oui & 45,0 & 41,7 & $>0,05$ \\
\hline \multirow{2}{*}{$\begin{array}{l}\text { Fumez-vous actuellement? } \\
(n=60)\end{array}$} & Non & 93,3 & 91,7 & $>0,05$ \\
\hline & Oui & 6,7 & 8,3 & $>0,05$ \\
\hline \multirow{3}{*}{$\begin{array}{l}\text { Accès à des professionnels de la santé } \\
(n=61)\end{array}$} & Neutre & 6,6 & 3,3 & $>0,05$ \\
\hline & En désaccord & 14,8 & 8,2 & $>0,05$ \\
\hline & D'accord & 78,7 & 88,5 & $>0,05$ \\
\hline \multirow{3}{*}{$\begin{array}{l}\text { Connaissance des programmes et des } \\
\text { services communautaires } \\
(n=61)\end{array}$} & Neutre & 14,8 & 11,5 & $>0,05$ \\
\hline & En désaccord & 24,6 & 11,5 & $>0,05$ \\
\hline & D'accord & 60,7 & 77,0 & $>0,05$ \\
\hline \multirow{3}{*}{$\begin{array}{l}\text { Compréhension de la façon } \\
\text { d'améliorer la santé } \\
(\mathrm{n}=61)\end{array}$} & Neutre & 6,6 & 3,3 & $>0,05$ \\
\hline & En désaccord & 16,4 & 6,6 & $>0,05$ \\
\hline & D'accord & 77,0 & 90,2 & $>0,05$ \\
\hline \multirow{3}{*}{$\begin{array}{l}\text { Relations avec des personnes du voisinage } \\
(n=59)\end{array}$} & Neutre & 13,6 & 11,9 & $>0,05$ \\
\hline & En désaccord & 27,1 & 16,9 & $>0,05$ \\
\hline & D’accord & 59,3 & 71,2 & $>0,05$ \\
\hline
\end{tabular}

\section{Leadership, structure et organisation}

Les groupes HANS étaient dirigés par les participants, mais les commentaires les plus courants concernaient le besoin de davantage de leadership et de structure. Un leader a émergé dans la plupart des groupes, mais tous les participants n'ont pas exercé leur leadership. Des groupes ont été déstabilisés lorsque le leader n’a plus été là pour diriger, et certains groupes ont perdu des membres en raison de l'attrition et de changements de dates ou de lieux de réunion. Certains groupes ont mieux réussi que d'autres à définir les types de séances éducatives qu'ils voulaient. Les participants désiraient plus d'interactions avec la personne-ressource de NorWest et ont suggéré plusieurs améliorations, comme des réunions régulières avec un programme prédéfini.
Accès

La participation régulière aux groupes HANS s'est révélée difficile pour certains participants. Ont été mentionnés des horaires chargés et d'autres responsabilités, ainsi que des difficultés liées au transport et au lieu de réunion. Les groupes en milieu de travail ont été confrontés à des difficultés spécifique en matière d'horaire de réunion. 
TABLEAU 4

Facteurs positifs et négatifs influençant la santé et citations représentatives des participants HANS

\begin{tabular}{|c|c|c|}
\hline & s ayant un effet sur la santé & Citations \\
\hline \multirow{7}{*}{$\begin{array}{l}\text { Facteurs } \\
\text { positifs }\end{array}$} & Relations de soutien & $\begin{array}{l}\text { « un soutien familial important », « mon mari », « mes enfants », « mes amis », « les membres de HANS ont } \\
\text { apporté leur aide », « [ils] jouent un rôle important », «j'adore leur parler et certains sont de bons amis, et } \\
\text { nous socialisons en dehors du groupe HANS KAII, il est bon de parler des choses et de partager » }\end{array}$ \\
\hline & Activité physique & $\begin{array}{l}\text { « activité physique régulière », « cours d’activité physique } 3 \text { fois par semaine », « hip hop », « Zumba », } \\
\text { « marcher », « jouer avec les enfants », « yoga » }\end{array}$ \\
\hline & Amélioration de l'alimentation & « changement dans l'alimentation », « consommer des aliments sains », « manger mieux » \\
\hline & Soutien communautaire & « rencontrer des gens », « interagir avec le groupe [HANS] m'aide... [les] relations que nous avons établies » \\
\hline & Bonne santé mentale & $\begin{array}{l}\text { « aimer mon travail », « changements à mon travail », « arrêter de travailler », « apprendre à gérer les } \\
\text { problèmes », « moins de stress » }\end{array}$ \\
\hline & Attitudes et croyances positives & « responsabilité envers le groupe », « motivé lorsque [je suis] avec les autres », « indépendant » \\
\hline & Accès aux services & $\begin{array}{l}\text { [Accès à] « médecin », « diététicien », « psychologue », « personnel infirmier », «j’ai une équipe mainte- } \\
\text { nant », « toutes sortes de spécialistes [NorWest] » }\end{array}$ \\
\hline \multirow{6}{*}{$\begin{array}{l}\text { Facteurs } \\
\text { négatifs }\end{array}$} & Mauvaise santé mentale et stress & $\begin{array}{l}\text { "je me sens submergé », « horaires chargés », « responsabilités professionnelles et problèmes avec... la } \\
\text { famille et les amis », « manque de soutien... [au travail] », "j'ai un travail très stressant », « je travaille trop } \\
\text { d’heures... j’ai trop de responsabilités », « la vie de famille est toujours stressante », « mon/ma/mes... [fils, } \\
\text { fille, mari, maman, enfants] », « décès dans la famille » }\end{array}$ \\
\hline & Mauvaise alimentation & $\begin{array}{l}\text { " assister à... des rassemblements », « aller manger au restaurant », « les vacances », « mauvaises habitudes } \\
\text { alimentaires », « [nous mangeons des aliments peu sains] quand je n'ai pas le temps », « être en surpoids » }\end{array}$ \\
\hline & Manque d'activité physique & $\begin{array}{l}\text { "pas assez de temps », « ne prends pas le temps [de pratiquer une activité physique] »; « douleur due à une } \\
\text { blessure », « les conditions météo » }\end{array}$ \\
\hline & Mauvaise santé & « maladie cardiaque », « diabète », « arthrite », « douleur » \\
\hline & $\begin{array}{l}\text { Manque de temps ou d'équilibre } \\
\text { entre le travail et la vie personnelle }\end{array}$ & $\begin{array}{l}\text { « exigences professionnelles », « exigences familiales », « simplement été très occupé... pas beaucoup de } \\
\text { temps pour moi » }\end{array}$ \\
\hline & Relations non favorables & « pas suffisamment de soutien de ma famille », « ma famille a rendu la situation plus difficile » \\
\hline
\end{tabular}

Le tableau 6 résume les améliorations suggérées pour le modèle HANS KAÏ, avec des citations représentatives.

FIGURE 5

Avantages de la participation au projet HANS (nombre de réponses)

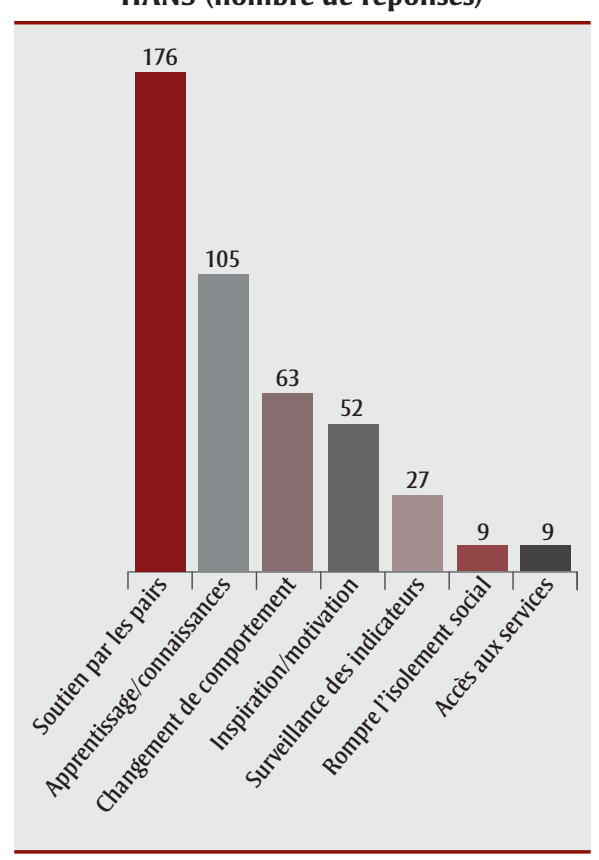

\section{Analyse}

Le but de cette étude était de déterminer si des groupes locaux structurés et dirigés par des pairs pouvaient être instaurés avec succès en contexte urbain canadien et avaient un effet positif sur la santé des participants. Une méta-analyse de 148 études a en effet révélé que les relations de soutien étaient corrélées à une diminution des risques de décès ${ }^{21}$. Berkman et Glass ${ }^{22}$ ont déterminé quant à eux que les adultes isolés socialement avaient un taux de mortalité deux à cinq fois plus élevé que les autres. Si le soutien par les pairs a été utilisé dans des initiatives de promotion de la santé, il l'a été surtout dans des initiatives de promotion de la santé ciblant certaines maladies en particulier ${ }^{23-25}$.

Nos résultats semblent indiquer que la participation à un groupe HANS a été bénéfique, notamment sur les plans suivants : soutien par les pairs, apprentissage et connaissances, changement de comportement, inspiration, motivation et prise de responsabilité, possibilité de rompre l'isolement social et accès accru aux soins primaires et à d'autres services liés à la santé. Ces résultats concordent avec des recherches antérieures sur les groupes de soutien par les pairs et leur effet sur la santé. Les approches dirigées par les pairs qui contribuent au sentiment d'" appartenance » locale ont un effet positif sur les « comportements de santé les plus importants (activité physique, perte de poids et régime alimentaire amélioré) »[traduction] ${ }^{26, \text { p. } 277 .}$ Selon un examen systématique de 25 essais contrôlés randomisés évaluant les changements de comportement chez des adultes plutôt âgés, les interventions par les pairs contribuent à un changement positif des comportements liés à la santé comme l'augmentation de l'activité physique, la diminution du tabagisme, l'utilisation accrue de préservatifs et l'augmentation du suivi de directives préalables ${ }^{27}$. Si la plupart des initiatives locales de soutien par les pairs ont ciblé un comportement spécifique en matière de santé, certaines ont été mises en œuvre pour créer un soutien social ou des réseaux sociaux en vue de prévenir l'isolement social ${ }^{2}$. Dans une étude de 2015 sur un programme communautaire visant la création de réseaux de soutien par les pairs, les principaux thèmes qui ont émergé ont été la création de réseaux sociaux, l'amélioration du bien-être et l'offre de services axés sur l'autonomisation ${ }^{28}$. Des études 
TABLEAU 5

Effets positifs sur la santé de la participation au groupe HANS et citations représentatives

Effets sur la santé de la participation au groupe HANS

Partage, écoute et soutien : « Nous avons beaucoup de problèmes similaires... c'est un soulagement de savoir que l'on n'est pas seul.... Mes problèmes... semblent bien plus gérables... S'ils peuvent le faire, je peux... aussi le faire »; «le projet HANS KAÏ m'a peut-être sauvé la vie », « je vais la chercher [pour aller à la réunion du groupe HANS] », « elle m'a conduit à mon rendez-vous chez le médecin », « je lui donne des livres de cuisine, parce qu'elle n'en a pas»

Soutien par les pairs Sentiment de sécurité et absence de jugement : « Si une personne aborde un problème, nous pouvons en parler... [sans] jugement... je me sens tellement soutenue », « si j'ai besoin d'aide, je n'ai pas peur d'en demander », « tout le monde a ses propres conflits et dans [cet] environnement, nous avons un endroit sûr pour parler de ces choses... »

Amitié et sentiment d'appartenance : « avoir l'impression de faire partie de la communauté », « ils sont mes amis maintenant », «nous nous rencontrons en dehors du groupe », « [les appels et les messages] aident à assurer le suivi »

Renseignements précis sur la santé : « manger sainement... collation... alimentation », « gestion de la glycémie », « lecture des étiquettes », Apprentissage et connaissances «j'obtiens des renseignements que j'utilise toujours »

Effet de l'apprentissage et des nouvelles compétences : «nous obtenons des renseignements et ensuite nous les mettons en pratique », « amener de nouvelles choses dans ma vie - c'est une bonne chose »

Alimentation : «je fais de meilleurs choix alimentaires », « maintenant je mange plus de fruits et de légumes », «j’ai réduit ma consommation de sel et j'achète des viandes maigres »

Exercice physique : «le groupe HANS m'a motivé à commencer à pratiquer la marche », «j'ai commencé à faire de l'exercice physique » Stress : « cela m’a permis de déterminer ce qui en est la cause [du stress] », «j'[ai utilisé] les techniques de libération du stress que j’ai appris dans le groupe de HANS KAÏ »

Changement de comportement

Inspiration/ motivation/ prise de responsabilité

Surveillance des indicateurs

Rompre l'isolement social

Accès aux services
Gestion du poids/de la glycémie/de la tension artérielle : «j'ai perdu du poids », « [depuis ma participation au groupe HANS] j’ai commencé à prendre mes médicaments régulièrement », " ma ǵlycémie est meilleure », " mon médecin a réduit mes... médicaments », « [mon médecin] a constaté une [amélioration] significative... par rapport à quand j'ai commencé le programme HANS... »

Établissement d'objectifs : «j'apprends comment définir des objectifs raisonnables », «je respecte [mes objectifs] »

Répercussions sur les autres : « lorsque vous achetez des aliments... vous devez consulter l'étiquette... j'apprends également aux membres de ma famille à [consulter les étiquettes] », «me fait penser [lorsque] je prépare... le repas pour mes enfants »

Autres changements : «j'ai arrêté de fumer », «je fais de meilleurs choix plus sains », «je ris plus »

Inspiration : « lorsque je vois d'autres personnes adopter un changement positif ou faire de gros efforts pour se sortir de l'impasse... cela m'inspire », « essayer de nouvelles choses est une source d'inspiration»

Motivation : «à cause d'eux, vous continuez de faire des choses que vous ne voulez pas faire », «motivation pour tenir bon »

Responsabilisation : «le groupe maintient ma responsabilité », «prendre [votre santé] en mains... [c'est] un état d'esprit qui est différent »

Partage des avantages : «j'encourage d'autres personnes à participer... “vous apprendrez quelque chose!” », «j'essaie d'inviter [d'autres personnes lorsque] nous avons des conférenciers particuliers... », « je partage les renseignements avec ma sœur et ma mère... [comme] de pratiquer une activité physique, manger sainement... »

Autosurveillance : «me permet de garder le contrôle... d'être vigilant », « je surveille... plus régulièrement... ma glycémie est devenue plus régulière », «je n'ai pas à... attendre le prochain rendez-vous »

Sentiment d'appartenance : «j'éprouve un sentiment d'appartenance», «je fais partie de quelque chose », «je n'ai pas beaucoup d'amis... il est agréable de savoir qu'il y a quelqu'un qui pense à vous [et se souvient de vous] », «j'ai vécu dans cette région depuis 30 ans, mais je n'ai commencé à avoir l'impression de faire partie de la communauté que depuis ma participation au groupe HANS KAÏ »

Sortir : « m'a aidé à... sortir », « amène les gens à sortir de leur isolement », « le projet nous force, nous les mères au foyer, à sortir... c'est probablement la meilleure chose...»

Services communautaires : « ils [NorWest] nous informent des services offerts dans la communauté », « si j’ai besoin de renseignements, je [sais qui] je peux appeler et c'est une bonne chose... »

Services de NorWest : «j'ai une diététiste [et] des soins des pieds... par le biais de NorWest... grâce au projet HANS KAÏ », « le soutien est vraiment bon entre NorWest et HANS KAÏ et [la façon dont ils] interagissent avec la communauté est vraiment importante », « NorWest nous offre un filet de sécurité » ont également permis de constater que le soutien par les pairs améliorait l'accès aux services de soins primaires, que ce soit de l'information sur la santé, des programmes communautaires ou des services de soutien $^{24,26,28-36}$. De plus, les interventions par les pairs ont permis des accomplissements communs, grâce à l'action, la présentation de modèles de rôle et l'échange de connaissances, ce qui a contribué à davantage de satisfaction, de confiance en soi et d'acceptation au sein des membres du groupe $e^{24,36-41}$.

Cette recherche vient enrichir la littérature relative aux effets sur la santé des groupes de soutien visant les comportements interdépendants de prévention des maladies chroniques (alimentation saine, activité physique régulière, suivi des indicateurs de santé et soutien social) dans le cadre d'un modèle de soutien par les pairs qui ne soit pas axé sur un seul problème de santé, une maladie chronique ou un comportement précis lié à la santé.

Bien que les avantages du soutien par les pairs soient bien décrits dans la littérature, on ne peut pas affirmer que les preuves empiriques soient sans équivoque sur cette question. Webel et ses collaborateurs ${ }^{27}$ ont 


\section{TABLEAU 6}

\section{Suggestions des participants pour améliorer le projet HANS KAÏ et citations représentatives}

\begin{tabular}{|c|c|}
\hline \multicolumn{2}{|r|}{ Améliorer le projet HANS KAÏ } \\
\hline \multirow{2}{*}{ Format des réunions } & $\begin{array}{l}\text { Fréquence et durée : «j’aimerais que nous puissions nous rencontrer plus souvent », «je pense que les réunions devraient être plus } \\
\text { longues », « je préfèrerais que nous ne nous réunissions [qu']une fois par mois » }\end{array}$ \\
\hline & $\begin{array}{l}\text { Membres : «nous ne sommes pas aussi nombreux que nous le voudrions », "si nous pouvions avoir quelques membres supplémentaires, } \\
\text { ce serait bien » }\end{array}$ \\
\hline \multirow{3}{*}{$\begin{array}{l}\text { Leadership, structure } \\
\text { et organisation }\end{array}$} & $\begin{array}{l}\text { Leadership : « ne pas avoir de personne responsable peut fonctionner au Japon, mais je ne pense pas que cela fonctionne ici », « avec une } \\
\text { rotation des leaders, il n'y a personne qui [coordonne] réellement le groupe », « nous avons__ elle assure la cohérence de l'ensemble. Ce } \\
\text { serait difficile si elle n'était pas [là]», « nous avions... une personne qui assumait plus de responsabilités [qui] est partie et depuis, le groupe } \\
\text { s'est quasi désintégré » }\end{array}$ \\
\hline & $\begin{array}{l}\text { Structure : « nous recevons différents conférenciers, ce qui est une excellente chose », "j'aimerais que [les présentateurs soient] mieux } \\
\text { préparés », " plus de sujets», " plus de discussions de groupe », " plus de cours d'activité physique », « ramener un financement pour les } \\
\text { collations» }\end{array}$ \\
\hline & $\begin{array}{l}\text { Organisation : « je mettrais vraiment l'accent sur... une structure mieux définie et plus de soutien de la part de la personne-ressource de } \\
\text { NorWest, « ils ne font qu'entrer et sortir », « [besoin] d'une vérification régulière » }\end{array}$ \\
\hline Accès & $\begin{array}{l}\text { Accès : «trouver du temps », « distance que je dois parcourir pour m’y rendre », « je ne peux pas y assister pendant la journée », « utiliser } \\
\text { un transport adapté... parfois je n'ai pas assez pour des billets supplémentaires », « horaires chargés », "c'est... après le travail » }\end{array}$ \\
\hline
\end{tabular}

procédé à un examen systématique de l'efficacité des interventions par les pairs en vue d'un changement de comportement précis et ont conclu que les preuves étaient mitigées. Certaines interventions étaient efficaces (activité physique, tabagisme et utilisation du préservatif), alors que d'autres ne l'étaient pas (allaitement maternel, observance thérapeutique et dépistage en matière de santé des femmes).

Les observations de notre étude sont similaires. Les résultats quantitatifs du sondage auprès des participants montrent que la participation à un groupe HANS a amené une amélioration statistiquement significative uniquement dans les scores en santé mentale, avec en outre des améliorations positives dans d'autres mesures liées à la santé. L'analyse thématique qualitative des entrevues en personne a révélé que la participation au groupe HANS avait eu un effet positif sur la santé des participants, principalement par le soutien des pairs, ainsi que sur l'apprentissage et l'accroissement des connaissances. De plus, les deux tiers des participants ont signalé un changement de comportement, même si aucun changement statistiquement significatif n'est ressorti des données quantitatives. Cela pourrait s'expliquer par la façon dont les questions sur le changement de comportement ont été présentées dans le sondage, ou cela pourrait indiquer que les méthodes qualitatives ouvertes rendent mieux compte des changements de comportement que les méthodes quantitatives. Nous avons été surpris de constater une absence de stress lié au revenu (ce que nous avions anticipé avec la question ouverte « qu'est-ce qui ne vous a pas aidé ou a nui à votre capacité à rester en santé et à vous sentir bien? »), malgré une proportion importante de participants au chômage ou relevant d'un groupe à faible revenu.

En résumé, les groupes HANS KAÏ semblent avoir un effet positif significatif sur la santé mentale des participants, comme le montrent les résultats quantitatifs comme qualitatifs. De plus, les participants ont bénéficié d'un soutien et de liens accrus, ce qui peut avoir des effets positifs sur certains aspects de leur santé, notamment des changements de comportement. La relation entre la participation à un groupe HANS KAÏ et l'amélioration d'indicateurs mesurables tels que la glycémie, la pression artérielle, le poids et le tour de taille est moins claire. Davantage de recherches sont nécessaires pour déterminer si la participation à un groupe HANS a un effet sur l'évolution de ces indicateurs.

\section{Limites}

Notre étude comporte plusieurs limites. Notre échantillon a été recruté dans une seule province canadienne et était principalement composé de femmes. Le manque de participation masculine pourrait être attribuable à l'hésitation des hommes à demander de l'aide pour des problèmes de santé, en particulier en ce qui concerne les interventions préventives, et nous offre de ce fait l'occasion d'examiner comment inclure plus d'hommes dans l'intervention. Ces limites peuvent restreindre la transférabilité des résultats. De plus, notre échantillon n'était pas de grande taille : les résultats sont fondés uniquement sur les groupes qui se réunissaient régulièrement et sur les commentaires autodéclarés. La méthode qualitative utilisée a cependant fourni des renseignements détaillés susceptibles de compenser cette limite. Nous n'avons pas pu mesurer l'évolution des indicateurs de la glycémie, de la pression artérielle, du poids et du tour de taille car les journaux de bord servant au suivi de ces indicateurs de santé automesurés se sont révélés incomplets. Les changements de comportement autodéclarés en matière de santé (généraux et particuliers) pourraient ne pas refléter la réalité mais correspondre à des biais de rappel ou de désirabilité sociale. En outre, quatre des groupes ont mis fin à leurs activités pour diverses raisons (perte d'intérêt et conflits d'horaire, entre autres). Certains participants ont manqué certaines réunions lors desquelles les entrevues de suivi ont eu lieu, n'étaient pas disponibles ou ont été perdus de vue, sans compter diverses difficultés quant à la participation régulière aux réunions. À cet égard, Gustavson et ses collaborateurs ont mentionné le défi des taux d'attrition élevés dans la recherche interventionnelle en santé publique (30 à $70 \%$ ), situation susceptible d'avoir une incidence sur la généralisabilité des résul$\operatorname{tats}^{42}$. Malgré les tentatives faites pour joindre les personnes ayant cessé de participer, les chercheurs n'ont pu réaliser que quelques entrevues de sortie.

Il existe des différences statistiquement significatives entre les caractéristiques individuelles des participants à la recherche et ceux perdus de vue, ce qui fait que 
l'absence de données sur les abandons pourrait avoir eu un impact sur les résultats de l'étude. Le modèle de soutien par les pairs utilisé dans cette étude impliquait intentionnellement une direction par des pairs, tous les participants constituant des pairs et les professionnels de la santé de NorWest fournissant conseils et soutien, mais les commentaires les plus fréquents sur l'amélioration du modèle HANS ont été des demandes pour plus de leadership et de structure.

Si le soutien par les pairs peut favoriser l'acquisition de connaissances, un sentiment d'appartenance et l'autogestion de la santé, d'autres méthodes individuelles ou collectives peuvent également se révéler efficaces, et les professionnels de la santé ont à savoir laquelle est susceptible de se révéler la plus adaptée à un client $\mathrm{t}^{43-44}$. Améliorer sa santé avec un groupe de soutien par les pairs n'est pas une stratégie adaptée ou efficace pour tout le monde, ce qui fait que le modèle de direction par les pairs, associé à une perception de manque de soutien, pourrait avoir contribué au taux d'attrition.

La littérature sur la promotion de la santé indique que les interventions axées sur le changement de mode de vie ou de comportement au niveau individuel peuvent avoir des effets limités à long terme car " les comportements liés à la santé sont influencés par de nombreux facteurs : les pressions culturelles, la littératie en santé, les inégalités en matière de santé, la capacité mentale, la prédisposition génétique et, dans le cas du tabagisme ou de l'alcool, la dépendance à la substance $»^{45, \text { p.1 }}$. Paradoxalement, une intervention de 12 à 24 mois est potentiellement trop courte pour étudier les effets durables sur la santé de la participation au groupe HANS.

\section{Conclusion}

Au fur et à mesure que l'on met l'accent en soins de santé sur la promotion de la santé plutôt que sur le traitement des maladies, l'utilisation du soutien par les pairs tend à se généraliser, non seulement en santé mais aussi en science du comportement ${ }^{2}$. La participation à un groupe HANS KAÏ, enchâssée à un modèle de soutien par les pairs, vise à soutenir la santé par diverses interventions en promotion de la santé comme l'éducation, l'action, un meilleur accès aux services et l'autonomisation. Notre étude, axée sur le contexte local, est motivée par le besoin de créer des moyens novateurs de prévention et de gestion des maladies chroniques au sein d'une population confrontée à des facteurs interdépendants (déterminants sociaux de la santé) tels qu'un faible niveau de scolarité et de revenu, l'isolement social et le manque d'accès à des services de santé et de loisirs.

Cette étude s'est déroulée en utilisant un modèle participatif réunissant NorWest et les chercheurs. Un conseil local et les résidents du quartier ont fourni une orientation et des commentaires. La durée de suivi des participants pour ce projet de recherche a pu atteindre 24 mois, mais la participation à un groupe HANS étant censée durer à long terme, il était prévu que les groupes poursuivent leurs activités bien au-delà de la fin du projet de recherche. Cette spécificité peut constituer une amélioration par rapport à d'autres interventions de prévention des maladies chroniques réduites à une durée prescrite (6 à 12 semaines) ou se concentrant sur une seule maladie chronique ou un seul changement de comportement.

Les résultats de cette recherche indiquent que le modèle HANS KAÏ a entraîné des améliorations statistiquement significatives en matière de santé mentale. Les résultats de l'analyse qualitative montrent que la participation à un groupe HANS a présenté d'autres avantages, notamment le soutien par les pairs, l'apprentissage et les connaissances, le changement de comportement, l'inspiration, la motivation et la prise de responsabilité, la possibilité de rompre l'isolement social et un accès accru aux soins primaires et à d'autres services liés à la santé.

Bien que d'autres recherches soient nécessaires pour valider ces résultats, il semble que l'approche HANS KAÏ, dans la mesure où elle va au-delà d'un changement de comportement individuel et où elle tient compte de l'importance de la collectivité locale, puisse constituer une approche efficace pour créer un environnement propice à l'entraide mutuelle pour favoriser des choix de vie sains et détecter rapidement des changements dans l'état de santé.

\section{Remerciements}

Le financement a été fourni par le NorWest Co-op Community Health Centre. Les auteures souhaitent remercier le personnel de NorWest et tous les participants des groupes HANS KAÏ qui ont participé aux recherches. Nous aimerions également remercier les organisateurs du projet HANS KAÏ de NorWest pour leur soutien et leur contribution, Nancy Heinrichs (directrice générale), Michelle Kirkbride (coordonnatrice de l'équipe de développement communautaire), Shannon Milks (coordonnatrice des soins primaires) et Mike Sadlowski (spécialiste de la promotion de la santé), Benita Cohen (conseillère en recherche qualitative), Nawal Lutfiyya (analyste de données - données quantitatives), le $\mathrm{D}^{\mathrm{r}}$ Lawrence Elliot (conseiller en recherche quantitative), Kristy Boyko, Kate Hodgson et Adebusola Adekoya (aide à la recherche) ainsi que le Manitoba Centre for Nursing and Health Research.

\section{Conflits d'intérêts}

Les auteures déclarent n'avoir aucun conflit d'intérêts.

\section{Contributions des auteures et avis}

AH et HW ont travaillé en étroite collaboration avec le groupe consultatif HANS KAÏ du NorWest Co-op Community Health Centre pour la conceptualisation, la planification et la réalisation de la recherche. D'autres contributeurs ont apporté un soutien supplémentaire (voir la section Remerciements). HW a joué un rôle primordial dans la conception et l'exécution du plan de projet, et $\mathrm{AH}$ a dirigé la collecte et l'analyse des données ainsi que la rédaction du rapport préliminaire. Les deux auteures ont lu et approuvé le manuscrit final.

Les auteures assument la responsabilité du contenu de l'article et des points de vue qui y sont exprimés. Ceux-ci ne reflètent pas nécessairement ceux du gouvernement du Canada.

\section{Références}

1. Mead S, Hilton D, Curtis L. Peer support: a theoretical perspective. Psychiatr Rehabil J. 2001;25(2):134-141.

2. Dennis CL. Peer support within a health care context: a concept analysis. Int J Nurs Stud. 2003;40(3):321-332.

3. Dennis CL. The process of developing and implementing a telephone-based peer support program for postpartum depression: evidence from two randomized controlled trials. Trials. 2014; 15(1):131. doi: 10.1186/1745-6215-15-131. 
4. Thomas GN, Macfarlane DJ, Guo B, et al. Health promotion in older Chinese: a 12-month cluster randomized controlled trial of pedometry and "peer support". Med Sci Sports Exerc. 2012;44(6):1157-1166. doi: 10.1249 /MSS.0b013e318244314a.

5. Ford P, Clifford A, Gussy K, et al. A systematic review of peer-support programs for smoking cessation in disadvantaged groups. Int $\mathrm{J}$ Environ Res Public Health. 2013;10:5507-22. doi: 10.3390/ijerph10115507.

6. Panayotof-Schaan L. An overview of health co-operatives: a case study perspective using Canadian and international examples. Victoria (BC) : British Columbia Institute for Cooperative Studies; 2009. En ligne à : http://planh.ca/sites/default/files /panayotof-schaan_healthcooperatives .pdf

7. Girard JP, Restakis J. To Life! Japan's model of co-operative health care \& what it means for Canada. Making Waves. 2008;19(1):5-8.

8. Bandura A. L'apprentissage social. Bruxelles (Belgique) : Mardaga; 1980.

9. Zimmerman MA. Empowerment theory. Dans : Rappaport J, Seidman E (dir.). Handbook of community psychology. New York : Kluwer Academy / Plenum Publishers; 2000. p 43-63.

10. Winnipeg Regional Health Authority; George and Fay Yee Centre for Healthcare Innovation, Evaluation Platform. Inkster community area profile, 2015. Winnipeg (MB) : Winnipeg Regional Health Authority; 2015. En ligne à : http://www.wrha .mb.ca/research/cha2014/files/Inkster .pdf

11. Kaplan and Associates. Inkster in action report. Winnipeg (MB): Kaplan and Associates; 2008.

12. Cresswell J, Klassen A, Clarke V, et al. Best practices for mixed methods research in the health sciences. Washington : National Institutes of Health, Office of Behavioral and Social Sciences Research (US); 2011. En ligne à : https://web.archive.org/web /20150920015338/https://www.nursing .virginia.edu/media/Best_Practices_ for_Mixed_Methods_Research.pdf
13. Government of Manitoba, Manitoba in Motion. Physical activity self-assessment. Winnipeg (MB) : Government of Manitoba; 2010.

14. Harland Gregoire MJ, Chow S, Spooner GR, et al. A screening instrument to identify adults with nutritional risks in a family medical practice. Can J Diet Pract Res. 1999; 60(4)205-211.

15. Government of Manitoba, Tobacco Control \& Cessation. Commit to quit self-assessment. Winnipeg (MB) : Government of Manitoba; 2010.

16. Williams MV, Baker DW, Parker RM, et al. Relationship of functional health literacy to patients' knowledge of their chronic disease. A study of patients with hypertension and diabetes. Arch Intern Med. 1998;158:166-172. doi: 10.1001/archinte.158.2.166

17. Hayes R, Stewart A. Sleep measures. In: Stewart A, Ware J, editors. Measuring functioning and well-being: the Medical Outcomes Study approach. Durham (NC): Duke University; 1992. p. 235-259.

18. Keyes C. Brief description of the mental health continuum short form (MHC-SF). Atlanta (GA): Emory University; 2009. En ligne à : https:// www.aacu.org/sites/default/files/MHC -SFEnglish.pdf

19. Ware JE Jr, Sherbourne CD. The MOS 36-item short-form health survey (SF36): I. Conceptual framework and item selection. Med Care. 1992;30(6): 473-483.

20. Gupta SK. Intention-to-treat concept: a review. Perspect Clin Res. 2011;2(3): 109-112. doi: 10.4103/2229-3485.83221.

21. Holt-Lunstad J, Smith T, Layton JB. Social relationships and mortality risk: a meta-analytic review. PLoS Med. 2010;7 (7):e1000316. doi: 10.1371 /journal.pmed.1000316.

22. Berkman LF, Glass TA. Social integration, social networks, social support, and health. In: Berkman LF, Kawachi I (dir.). Social epidemiology. New York : Oxford University Press; 2000. p. 137-173.
23. Bagnall AM, South J, Hulme C, et al. A systematic review of the effectiveness and cost-effectiveness of peer education and peer support in prisons. BMC Public Health. 2015;15:290. doi: 10.1186/s12889-015-1584-X.

24. Woolhouse S, Cooper E, Pickard A. "It gives me a sense of belonging": providing integrated health care and treatment to people with $\mathrm{HCV}$ engaged in a psycho-educational support group. Int J Drug Policy. 2013; 24(6):550-557. doi: 10.1016/j.drugpo .2013 .05 .018

25. Zhong X, Wang Z, Fisher EB, et al. Peer support for diabetes management in primary care and community settings in Anhui Province, China. Ann Fam Med. 2015;13 Suppl 1:S5058. doi: 10.1370/afm.1799.

26. Hystad P, Carpiano RM. Sense of community-belonging and healthbehaviour change in Canada. J Epidemiol Community Health. 2010; 66(3):277-283. doi: 10.1136/jech.2009 .103556

27. Webel AR, Okonsky J, Trompeta J, et al. A systematic review of the effectiveness of peer-based interventions on health-related behaviors in adults. Am J Public Health. 2010;100(2):247253. doi: 10.2105/AJPH.2008.149419.

28. Liamputtong P, Koh L, Wollersheim D, et al. Peer support groups, mobile phones and refugee women in Melbourne. Health Promot Int. 2016; 31(3):715-724. doi: 10.1093/heapro /dav015.

29. Aschbrenner KA, Naslund JA, Shevenell M, et al. Feasibility of behavioral weight loss treatment enhanced with peer support and mobile health technology for individuals with serious mental illness. Psychiatr Q. 2016;87(3):401-415. doi: 10.1007/s11126-015-9395-x.

30. Coşkun A, Karakaya E. Supporting safe motherhood services In Diyarbakir: a community-based distribution project Matern Child Health J. 2013;17(6): 977-988. doi: 10.1007/s10995-012-1102-z. 
31. Crawford S, Bath N. Peer support models for people with a history of injecting drug use undertaking assessment and treatment for hepatitis C virus infection. Clin Infect Dis. 2013;57 Suppl 2:S75-79. doi: 10.1093/cid /cit297.

32. Hanson LC, Armstrong TD, Green MA, et al. Circles of care: development and initial evaluation of a peer support model for African Americans with advanced cancer. Health Educ Behav. 2013;40(5):536-543. doi: 10.1177 /1090198112461252.

33. Hanson LC, Green MA, Hayes M, et al. Circles of care: implementation and evaluation of support teams for African Americans with cancer. Health Educ Behav. 2014;41(3):291-298. doi: 10.1177 /1090198113512127.

34. Mason K, Dodd Z, Sockalingam S, et al. Beyond viral response: a prospective evaluation of a community-based, multi-disciplinary, peer-driven model of HCV treatment and support. Int J Drug Policy. 2015;26(10):1007-1013. doi: 10.1016/j.drugpo.2015.04.012.

35. Rempel LA, Moore KC. Peer-led prenatal breast-feeding education: a viable alternative to nurse-led education. Midwifery. 2012;28(1):73-79. doi: 10.1016/j.midw.2010.11.005.

36. Swarbrick MA. Integrated care: wellness-oriented peer approaches: a key ingredient for integrated care. Psychiatr Serv. 2013;64(8):723-726. doi: 10.1176 /appi.ps.201300144.

37. Ashing-Giwa K, Tapp C, Rosales M, et al. Peer-based models of supportive care: the impact of peer support groups in African American breast cancer survivors. Oncol Nurs Forum. 2012;39(6):585-591. doi: 10.1188/12 .ONF.585-591.

38. Coniglio FD, Hancock N, Ellis LA. Peer support within Clubhouse: a grounded theory study. Community Ment Health J. 2010;48(2):153-160. doi: 10.1007/s10597-010-9358-5.

39. Lu Q, You J, Man J, et al. Evaluating a culturally tailored peer-mentoring and education pilot intervention among Chinese breast cancer survivors using a mixed-methods approach. Oncol Nurs Forum. 2014;41(6):629-637. doi: 10.1188/14.ONF.629-637.
40. Raine P. Promoting breast-feeding in a deprived area: the influence of a peer support initiative. Health Soc Care Community. 2003;11(6):463-469. doi: 10.1046/j.1365-2524.2003.00449.x.

41. Repper J, Watson E. A year of peer support in Nottingham: lessons learned. Journal of Mental Health Training, Education and Practice. 2012;7(2):7078. doi: 10.1108/17556221211236466.

42. Gustavson K, von Soest T, Karevold E, et al. Attrition and generalizability in longitudinal studies: findings from a 15-year population-based study and a Monte Carlo simulation study. BMC Public Health. 2012;12:918. doi: 10.1186/1471-2458-12-918.

43. Sav A, McMillan Sara, Kelly F, et al. Consumer health organisations for chronic conditions: why do some people access them and others don't? Prim Health Care Res Dev. 2014;15:112. doi: 10.1017/S1463423614000036.

44. Lawn S, Schoo A. Supporting selfmanagement of chronic health conditions: common approaches. Patient Educ Couns. 2010;80(2):205-211. doi: 10.1016/j.pec.2009.10.006.

45. Thirlaway K, Davies L. Lifestyle behaviours and personal responsibility for health [résumé]. eLS [Internet]. 2013. doi: 10.1002/9780470015902 .a0025159/abstract. 